\title{
Drug Supply Chain Management and Implementation of Health Reform Plan in Teaching Hospital Pharmacies of Ahvaz, Iran
}

\author{
Effat Jahanbani', Reza Shakoori', Masoume Bagheri-Kahkesh ${ }^{2 *}$ \\ ${ }^{1}$ Department of Health Services Management, School of Health, Ahvaz Jundishapur University of Medical Sciences, Ahvaz, Iran \\ ${ }^{2}$ Department of Management Development and Resources, Alborz University of Medical Sciences, Karaj, Iran
}

\begin{abstract}
*Corresponding Author: Masume Bagheri-Kahkesh, PhD Student in Health Care Management, Department of Management Development and Resources, Alborz University of Medical Sciences, Karaj, Iran. Tel: +98-9169812189; Fax:+986143269652; Email: masume.bagheri@yahoo.com
\end{abstract}

Received July 21, 2016; Accepted September 3, 2016; Online Published December 18, 2016

\begin{abstract}
Background: Drugs play a strategic role as a health commodity. Thus, the supply chain management of drugs is an important issue to reducing costs and thereby improving patient health.

Objective: This study evaluated the status of drug supply chain management and health reform in the pharmaceutical sector of teaching hospitals in Ahvaz, Iran.

Methods: This cross-sectional study was carried out in pharmacies of teaching hospitals in Ahwaz, Iran in 2015. Data was collected through a checklist extracted from the Evaluation Checklist of the Food and Drug Deputy and related articles. The study checklist examined six dimensions of the Evaluation Checklist, including drug storage, administrative regulations, preparation of medicines, drug distribution, taking medication, and implementation of health reforms in the pharmaceutical sector. The content validity of the checklist was confirmed by experts and pharmaceutical specialists. Data was analyzed for descriptive characteristics such as frequency and percentage using Excel version 2010 software.

Results: The findings show that hospitals met standards for administrative regulations with $78.5 \%$ and preparation of medicines with $77.25 \%$. The dimensions of drug storage with $74.75 \%$, taking medication with $74.25 \%$, implementation of health reforms with $71.5 \%$, and drug distribution with $62.5 \%$ were in a near-standard state.

Conclusion: Supply chain management systems of medicines in teaching hospitals are near-standard. To improve hospitals, offering proper training to employees, using the fixed-rate shopping system, and providing facilities to patients are recommended.
\end{abstract}

Keywords: Pharmacy, Organization and administration, Hospitals, Health reform

\section{Background}

Undoubtedly the status of health is one of the most important indicators of social development and progress. The provision of healthcare is also a major challenge to human life, and the treatment of disease is considered one way to achieve community health. ${ }^{1}$ The management of the drug circulation process to advance the aims of healthy people for sustainable development is an important issue. Each country should have a national drug policy as a main part of its health society policy so as to ensure the provision of effective, safe, qualified, and affordably-priced drugs for the government and people. ${ }^{2}$ Correcting the drug policies of developing countries is a main concern in the healthcare sector, and the World Bank and the World Health Organization (WHO) are in agreement about the necessity of reorganizing it. ${ }^{3}$ In developing countries, most imported drugs are expensive, and this increases the allocated exchange rate for drug imports and increases costs. Additionally, the resources available in health centers are often not properly used, and in most cases, lack of different resources gathered together, the squandering and waste of resources can be observed. ${ }^{4}$

According to the President of the Islamic Republic of Iran, Doctor Hassan Rouhani, reforms in the country are being run as the health development plan. The health development plan is a project that improves the way people live in the area of healthcare. In this project, it is hoped that a lot of people problems, including the cost to treat diseases, dissatisfaction with medical centers, time wasted waiting for treatment, etc. will be resolved; troubleshooting is the main advantage of this project. ${ }^{5}$ Financial challenges in hospitals, in other words reducing costs and increasing

Copyright (C) 2016 The Hospital Practices and Research. This is an open-access article distributed under the terms of the Creative Commons Attribution License (http://creativecommons.org/licenses/by/4.0), which permits unrestricted use, distribution, and reproduction in any medium, provided the original work is properly cited. 
revenue, are of great importance. After legal personnel costs, the hospital pharmacy spends $20 \%$ of the total budget, making up the largest percentage of the health budget. ${ }^{6}$ Injuries caused by medical errors occur in over $42 \%$ of patients, which contribute significantly to increasing health problems, social problems, and the healthcare costs of people and the government.

Among these factors are the roles of people as the main component of the drug culture and doctors and medical personnel as a cultural element. Disregard for this issue is followed by undesirable consequences, such as patient and caregiver dissatisfaction, the ruination of the country's healthcare systems, prolonged illness periods, medication side effects experienced by patients, diminished doctorpatient relationships, and increasing healthcare costs and drug problems.

\section{Objective}

Considering all the above-mentioned factors, this study examined the management of the drug supply chain and the implementation of a health development plan in pharmacies at teaching hospitals in Ahvaz, Iran.

\section{Methods}

This was a cross-sectional and usability study. The study population and hospital environment consisted of the committee members of drugs and treatment in the medical teaching hospitals of Ahvaz University of Medical Sciences, which included Shafa, Razi, and Imam Khomeini hospitals. All of the population entered in the study. The data collection tool for this research was a checklist developed from the checklist of the food and drug departments of the evaluated pharmaceutical sector of hospitals ${ }^{5}$ and related articles. ${ }^{2,3}$ The reliability and validity of the checklist were confirmed with a score of 0.79 using Cronbach $\alpha$ and by experts and the Pharmaceutical University of Jundishapur, respectively. Data was collected through checklists completed through interviews and direct observations. The checklist contained 72 questions regarding 6 dimensions based on the objectives of the study. The first dimension contained 21 questions regarding drugstores; the second through sixth dimensions contained 5 questions about drug preparation, 9 questions about drug consumption, 10 questions related to drug distribution, 9 questions related to the implementation of healthcare development plans in the pharmaceutical sector of hospitals, and 18 questions about the administrative regulations of medication in the hospital, respectively (Figure 1).

Checklists were completed by technical officials, hospital drug store officials, and researchers based on their observations. The questions could be answered yes or no and were designed for the assessment of drug chain management based on the planned checklist. Based on divisions by the author in each dimension, if more than $75 \%$ of the questions were answered yes, then the situation was standard, from $51 \%$ to $75 \%$ indicated a near-standard situation, and if $50 \%$ or less of the questions were answered

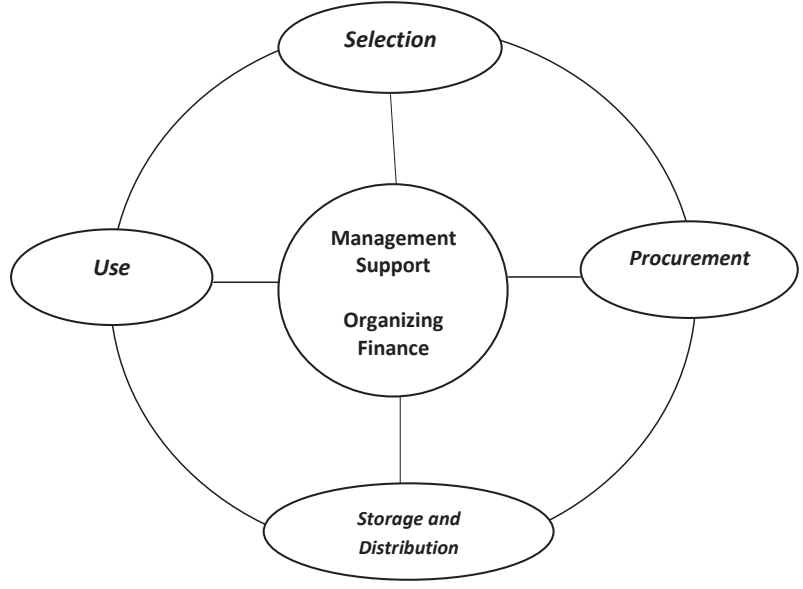

Figure 1. Medicine Chain Management Concept Model.

yes, the situation was considered to be substandard. Data was analyzed using Excel version 2010.

\section{Results}

The results demonstrated that the drugstores of all studied hospitals were in a standard situation in terms of software and hardware (computers, printers, hygrometer), proper shelving, and storage of medicines and serums according to the standards promulgated by the Ministry of Health. The situations of refrigerated storage of medication, security systems (fire-fighting and fire alarms, anti-theft systems), the presence of air conditioning and sanitation also met standard requirements. About $75 \%$ of the hospital pharmacies did not have a physical space proportional to the number of hospital beds. Additionally, expired drugs were kept in all pharmacies, and in only two of the hospitals (Shafa and Imam Khomeini) were reasons for drugs expiring examined by the Drugs and Drug Treatment Committee.

All hospitals met standards for the preparation of medicines in pharmacies, except the Imam Khomeini hospital in which conditions were near-standard. Seventyfive percent of the studied hospitals distributed drugs as single doses, and drugs outside the pharmacopoeia were provided for all hospitals under the prescription of the physicians, This is why patients were not sent out of the hospital in order to provide their drugs. All hospitals and pharmaceutical companies had a Committee of Medication and Treatment and advertising or contract systems were not used for drug purchasing. No independent financial relationship between pharmacies and pharmaceutical companies existed in any of the studied hospitals. All hospitals other than Imam Khomeini hospital met the standards for drug consumption. In $75 \%$ of hospitals, doctors and the pharmacies of the hospitals consulted with each other for some drugs, especially new ones. In all hospitals, communication existed between departments and pharmacies regarding drug consumption, prediction of the needed amount of drugs (Tralee and hospital emergency departments), sending invocations from departments to pharmacy stores with a mechanized method, and returning 
Table 1. Medicine Chain Management in Hospitals

\begin{tabular}{|c|c|c|c|c|}
\hline & Golestan Hospital & Shafa Hospital & Imam Khomeini Hospital & Razi Hospital \\
\hline Drug store state (\%) & 77 & 78 & 76 & 76 \\
\hline Drug provision (\%) & 80 & 80 & 60 & 80 \\
\hline Drug consumption (\%) & 88 & 77 & 44 & 88 \\
\hline Drug distribution (\%) & 70 & 52 & 40 & 60 \\
\hline Drug implementation criteria (\%) & 77 & 66 & 66 & 77 \\
\hline Implementation of health development plan (\%) & 88 & 72 & 77 & 77 \\
\hline
\end{tabular}

medicine that was close to the expiration date. In none of the hospital pharmacies except that of Imam Khomeini Hospital, was the accuracy of the prescribed dose for the patient examined. The stock of the departments was consistent with the checklist of pharmacies in $50 \%$ of the hospitals, and $75 \%$ of transactions between departments were recorded in an emergency situate ion in hospital pharmacies.

The Drug Distribution Departments of Golestan, Razi, and Shafa hospitals were in a near-standard situation, but Imam Khomeini hospital was substandard. Seventy-five percent of hospital pharmacies controlled the dispensing of medicines as quality, but priority was observed in Iran's drugs (according to the Food and Drug Department). In $50 \%$ of pharmacies, required actions were taken to deal with the problem of drugs being 2-tiered. In $75 \%$ of the pharmacies, there were drug savings for emergencies and critical situation proportional to the requirements. However, drugs were not distributed in the pharmacies based on the single-dose system. Moreover, drug distribution from stores to departments within 24 hours was not possible. Doctors of all hospitals were aware of the drugs available in the pharmacies, and the drugs requested by all departments were consistent with patient records and medical prescriptions. Seventy-five percent of the hospitals used the required facilities for transport, and in all hospitals, drug requests from departments were delivered to the pharmacy in certain hours and with stamp and complete signatures.

The implementation status of medical criteria was standard in all hospitals except Shafa hospital, in which conditions were near-standard. Retraining and training medical personnel were implemented in $50 \%$ of hospitals, while in half of the hospitals drug services were carried out 24 hours a day. In $75 \%$ of hospitals, there were enough pharmacists, and medical records for patients were kept in $50 \%$ of hospitals. In 3 hospitals, monthly reports on the drug status were presented orally to the head of the hospital. Technical personnel monitored the circulation of the drugs in all hospitals. In 75\% of hospitals, the side effects of consumed drugs were reported to the Hospital Pharmaceutical Committee. In all hospitals, the pharmacy technical personnel monitored the implementation of the Food and Drug Department rules. An updated pharmacopoeia was found in 3 of the 4 studied hospitals. In all hospitals, drugs were classified based on three categories (emergency, urgent, normal), and treatment committee and medication committee meetings were held regularly. In only one hospital were medication errors studied and reported on a regular basis.

Golestan and Razi hospitals were at a standard level for the implementation of health development plans in the hospital pharmaceutical sectors, and Imam Khomeini and Shafa hospitals were near-standard. Drugs from outside the pharmacopoeia that were emphasized and requested by physicians were found in the drug stores of half of all hospitals. All hospitals had a drug monitoring system in place for placing pharmaceutical requests to pharmaceutical distribution companies. In $75 \%$ of the studied hospitals, the insufficiencies of medicines and supplies were timely evaluated and essential drugs lists were compiled to preclude patients from going outside the hospital to find the required drug, even if it was not included in the hospital pharmacopoeia. In all studied hospitals, medication was prescribed based on early and final diagnoses. None of the hospitals provided health supplies for patient caregivers, despite the fact that all hospitals provided toiletries for admitted patients (shampoo, slippers, toothbrush, and toothpaste). None of the hospitals received the costs for prescribed drugs during treatments from patients. This is while at discharge, the cost of the prescribed drugs were received from patients according to the new tariffs of the health development plan (6\% urban insurance and 3\% referral rural insurance) (Table 1). The overall Medicine Chain Management in the study hospitals is shown in Figure 2.

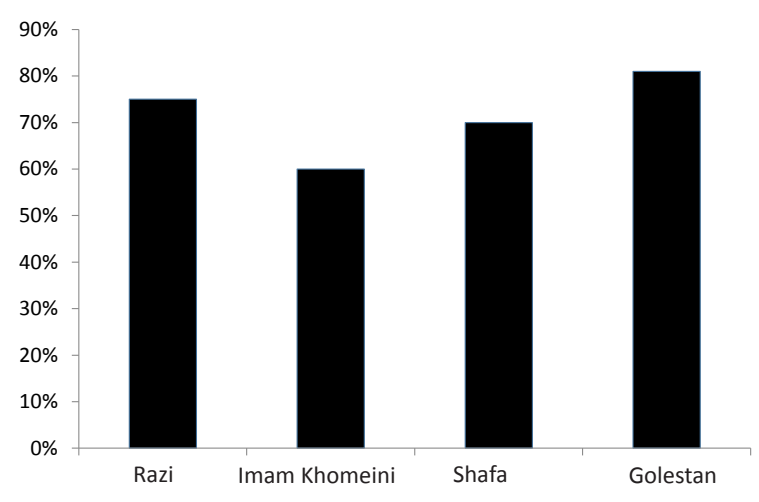

Figure 2. The Overall Status of Medicine Chain Management in the Study Hospitals. 


\section{Discussion}

The results of the present study showed that the pharmacies of all studied hospitals were in a standard condition and were consistent with Dinarvand's study on the necessity of education for all medical staff, especially hospital pharmacy personnel to minimize the risk of errors and maximize utilization of the equipment. ${ }^{9}$ Inappropriate and insufficient physical space in drugstores has caused many problems for commuting pharmacy staff, drug storage, warehousing, and inspection; it has also led to a buildup of drugs in the warehouse shelves that creates irreparable damages for the pharmacy. By insuring pharmacies and drug warehouses against unanticipated and anticipated risks (fires, earthquakes, floods, etc.) we can lead to providing the costs of adverse incidents through insurance organizations and the government and can somewhat guarantee pharmacies. The presence of stocks of expired drugs can be dangerous; this issue corresponds somewhat with the results of Berdot et al study. ${ }^{6}$

The results for drug provision showed that three of the four studied hospitals met standard levels, while Imam Khomeini hospital was near-standard. Some drugs were provided from pharmaceutical companies by the Food and Drug Department, and no independent financial relationship existed between the pharmacists and the pharmaceutical companies. Additionally, all of the studied pharmacies had a committee called the Medicine and Treatment Committee. This in itself is interesting, because this committee could provide expensive drugs that are not available in pharmacies or are not easily dispensed by pharmaceutical companies to pharmacies, thereby preventing the waste of time by drugstores. Another benefit observed in these pharmacies is the pharmacopoeia of drugs prepared for patients which saves patient from having to refer outside the hospital for drugs. This dimension was consistent with the findings of Berdot et $\mathrm{al}^{6}$ and Colella et al. ${ }^{4}$ In terms of drug distribution, the results showed that three of the four hospitals were in a standard state, while Imam Khomeini hospital was substandard. Drug distribution should be carried out by pharmacies (24-hour) according to the single-dose system.

Additionally, retraining and job training classes should be held for hospital pharmacy personnel so they become familiar with the methods and principles of drug usage, drug distribution, and drug services, including the principles of distribution and consumption of new and expensive drugs. Because excessive drug consumption reduces the desired effects of drugs on the consumer, the counsel for patients can prevent the harmful effects of excessive drug consumption. Studies have shown that between $1.5 \%$ and $43.5 \%$ of patients admitted to hospitals and $2 \%$ to $50 \%$ of outpatients in hospitals suffer from adverse effects induced by medication errors. Barnett concluded that the lack of monitoring in the distribution and consumption of drugs causes prolongation of hospitalization, ${ }^{10}$ and that complications from erroneous drug administration can be avoided with proper planning, timely and regular coordination with departments, and continuous communication with doctors and hospital sectors.

The findings revealed that Golestan and Razi hospitals met standards for health development plans, and Shafa and Imam Khomeini hospitals were near-standard. Implementation of a health development plan caused a reduction in medical expenses paid by people in hospitals and includes all areas of health and medicine. These results were consistent with those of a study by Bagheri Kahkesh et al. ${ }^{11}$ Another effect the health development plan had on pharmacies was the formation of a committee to assess drug costs in order to monitor induced costs, and to prevent patients from referring outside the hospital for medicine. This result was consistent with the findings of Chandani et al. ${ }^{12}$

\section{Conclusion}

It seems that the most important future challenges faced by pharmacies regarding health development plan management may include shortages of space due to the overcrowding of patients and the availability of excessive amounts of drugs both in pharmacies and stacked on warehouse shelves. Another challenge could be the shortage of trained manpower in pharmacies in order to help and advice those patients who refer to them. Another problem is excessive consumptions of drugs by patients. All these challenges and many more should be paid attention to so that it would eventually lead to less consumptions of drugs by patients.

\section{Authors' Contributions}

All authors contributed equally to this article.

\section{Conflicts of Interest Disclosures}

The authors had no conflicts of interest.

\section{Ethical Approval}

Not applicable.

\section{Acknowledgments}

The authors express their appreciation to their colleagues in the teaching hospitals of Ahvaz, Iran for their cooperation in the implementation of the plan.

\section{References}

1. Rahimikalamrudi $\mathrm{H}$. Reviews the drug system of health center of Guilan and providing ways to it's improve [Thesis]. Tehran: Tehran University of Medical Sciences; 1997. [Persian].

2. Fattahzadeh A. Health System Reform. 2nd ed. Tehran: Cultural Institute of Ibne Sina; 2006.

3. World Health Organization, The Nigerian Federal Ministry of Health. Baseline Assessment of The Nigerian Pharmaceutical Sector. http://apps.who.int/iris/bitstream/10665/69108/1/ a87426_eng.pdf. Published 2002.

4. Colella SJ, Lawrence SM, Widenhofer GJ, inventors; Pyxis Corporation, assignee. System of drug distribution to health care providers. United States patent US 6003 006. 1999 Dec 14. 
5. Jahangiri K. Principles of Disaster Management. Tehran: Tehran University of Medical Science; 2010.

6. Berdot S, Sabatier B, Gillaizeau F, Caruba T, Prognon P, Durieux P. Evaluation of drug administration errors in a teaching hospital. BMC Health Servs Res. 2012;12:60.

7. World Health Organization. Handbook for Drug Supply Management at the First-Level Health Facility. Geneva: WHO; 2006.

8. World Health Organization. Management of Drugs at Health Center Level. Geneva: WHO; 2004. http://apps.who.int/ medicinedocs/pdf/s7919e/s7919e.pdf.

9. Dinarvand R. Evaluation and the model presentation of regional distribution of drugs in Iran. Journal of Medical Council of Iran.
2005.

10. Barnett JW. Supply of Medicines Text Book of Hospital Pharmacy. London: Blackwell; 1980.

11. Bagheri Kahkesh M, Jahangiri K, Hajinabi K. Situational analysis of drug chain management in rural health houses at Masjed Soleiman district and effect of an educational intervention on process improvement. Journal of Healthcare Management. 2011;3(1-2):25-33.

12. Chandani Y, Felling B, Allers C, Alt D, Noguera M. Supply chain management of antiretroviral drugs: considerations for initiating and expanding national supply chains. Arlington, Va: DELIVER, for the U.S. Agency for International Development; 2006. 